\title{
Large B-cell lymphoma: a unique case with an insertional translocation of the entire MYC locus
}

\begin{abstract}
Follicular lymphoma is characterized by the $\mathrm{t}(14 ; 18)(\mathrm{q} 32 ; \mathrm{q} 21)$ translocation involving the BCL-2 and IGH genes. Aggressive double-hit lymphomas can arise from follicular lymphoma and are defined by a combination of a chromosomal breakpoint affecting MYC with one or more additional lymphoma associated abnormalities. We present a case of a 66year-old woman with a history follicular lymphoma who developed a large B-cell lymphoma with a novel Insertional translocation of the entire MYC locus from the $8 \mathrm{q} 24.1$ region into the derivative chromosome 14 of the $\mathrm{t}(14 ; 18)$ translocation. Additionally, the fusion IGH and BCL2 from the derivative chromosome 14 moved to same chromosome 8 at $8 \mathrm{q} 24.1$. To our knowledge, this type of insertion of the entire MYC locus into der(14) of the $\mathrm{t}(14 ; 18)$, and subsequent movement of the fused IGH/ BCL2 into 8q24.1, has not been previously reported.
\end{abstract}

Keywords: follicular lymphoma, large B-cell lymphoma, MYC, IGH, BCL-2, translocation, cytogenetic, immunohistochemistry, chromosome, hemolysis, lambda staining, follicular lymphoma
Volume 2 Issue | - 2016

\section{Ariana Geromes, Tori Leftwich, Kelley Mast, Ashwini Yenamandra}

Pathology, Microbiology and Immunology, Vanderbilt University School of Medicine, USA

Correspondence: Ariana Geromes, Vanderbilt University School of Medicine, Department of Pathology, Microbiology, and Immunology, MCN C-3324, I I6I 2 Ist Ave. South, Nashville, TN 37232-2561, USA, Email Ariana.Geromes@vanderbilt.edu

Received: December 12, 20I5 | Published: January I2, 2016
Abbreviation: Der(14), derivative chromosome 14; FISH, fluorescence in situ hybridization hyper; CVAD, chemotherapy with cyclophosphamide vincristine doxorubicin and dexamethasone

\section{Introduction}

Follicular lymphoma is characterized by the $\mathrm{t}(14 ; 18)(\mathrm{q} 32 ; \mathrm{q} 21)$ translocation leading to the juxtaposition of the $B C L-2$ proto-onco gene with the IGH promoter causing deregulation and over expression of this anti-apoptotic molecule. Additional cytogenetic abnormalities are often present in these malignancies and further aberrations can accrue and lead to the development of higher grade B-cell lymphomas. These high-grade lymphomas often display complex cytogenetic abnormalities including rearrangements of MYC. ${ }^{1}$ Double-hit lymphomas can arise from follicular lymphoma ${ }^{2}$ and are defined by a combination of a chromosomal breakpoint affecting the MYC locus with one or more additional lymphoma associated abnormalities, frequently $\mathrm{t}(14 ; 18){ }^{3}$ These malignancies have an aggressive clinical course and are typically resistant to chemotherapy. We present a case of a novel MYC rearrangement arising in a background of follicular lymphoma.

\section{Case presentation}

A sixty-six year old woman with a history of low grade follicular lymphoma, initially diagnosed in 2005 with bone marrow involvement, presented for evaluation of fever, hemolysis and thrombocytopenia in November of 2015. Her bone marrow biopsy showed extensive replacement by a population of large, atypical B-cells with variable forward scatter and the following abnormal immunophenotype by flow Cytometry: negative for significant kappa or lambda staining; positive for CD10, CD19 (bright), CD38, CD45, and cytoplasmic CD79a (heterogeneous dim); negative for CD5, CD20, CD23, CD34, CD200, and TdT. Increased expression of BCL-2 and MYC was demonstrated by immunohistochemistry. Cytogenetics was highly abnormal in 18 out of the 20cells with a complex karyotype: $50, \mathrm{XX},+\mathrm{X},+\mathrm{X},+1, \operatorname{der}(1 ; 15)(\mathrm{q} 10 ; \mathrm{q} 10),+7, ? \mathrm{t}(8 ;[\operatorname{der}(14) \mathrm{t}(14 ; 18)$ $(\mathrm{q} 32 ; \mathrm{q} 21.3)])(\mathrm{q} 24 ; \mathrm{q} 32),+12, \mathrm{t}(14 ; 18)(\mathrm{q} 32 ; \mathrm{q} 21.3)$, (Figure 1). FISH studies performed on the same specimen revealed a $\mathrm{t}(14 ; 18)$ in $87 \%$ of the cells. Metaphase FISH analysis of $\mathrm{t}(14 ; 18)$ and MYC slides revealed that the derivative chromosome $14(\operatorname{der}(14))$ appeared to be involved in an additional rearrangement with the MYC locus at 8q24. Directed metaphase FISH studies indicated that a portion of the der(14), including the IGH/BCL2 fusion signal, moved to the $8 \mathrm{q} 24$ region and the entire MYC locus from the same chromosome 8 was inserted into the der(14) (Figure 2). Additional probes detected a residual IGH signal on the der(14). No residual MYC signal at 8q24 was detected; instead, a fusion of the entire MYC locus with the residual IGH was observed on the der(14) (Figure 3). Based on these findings, it is unclear if the IGH/BCL-2 fusion localized at 8q24 is fused with a residual portion of the MYC locus resulting in a complex $\mathrm{MYC} / \mathrm{IGH} / \mathrm{BCL}-2$. It is also not clear if this complex rearrangement involves additional genes. The patient underwent one cycle of chemotherapy with cyclophosphamide, vincristine, doxorubicin, and dexamethasone (Hyper CVAD) shortly after diagnosis. She was subsequently discharged with outpatient follow up.

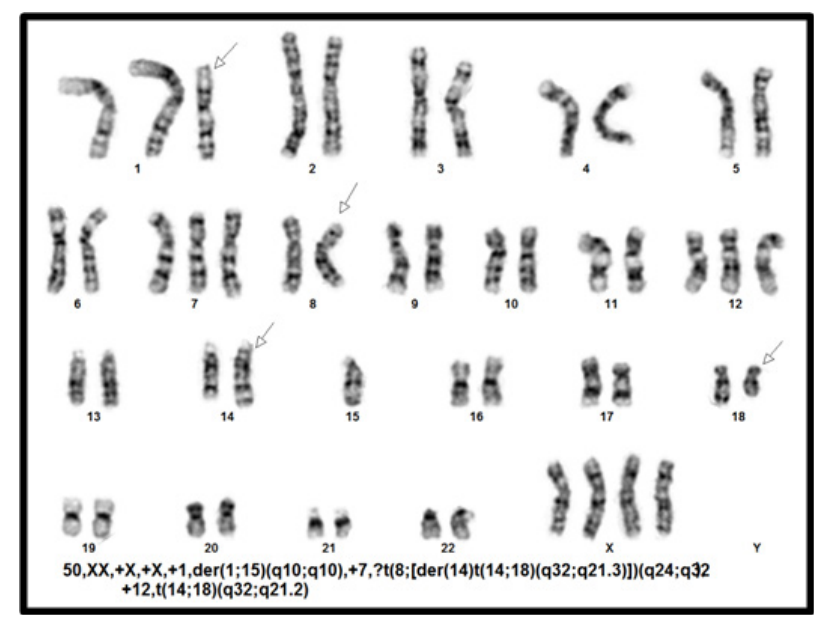

Figure I The patient's complex karyotype. 


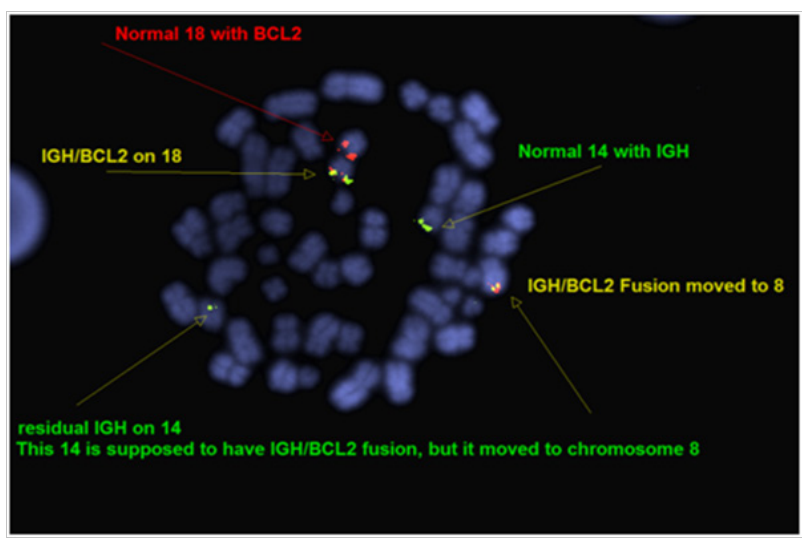

Figure $2 \mathrm{~A} t(14 ; 18)$ probes show two IGH/ BCL-2 fusion signals (yellow). One of these fusion signals has translocated to chromosome 8; the other remains on chromosome 18.

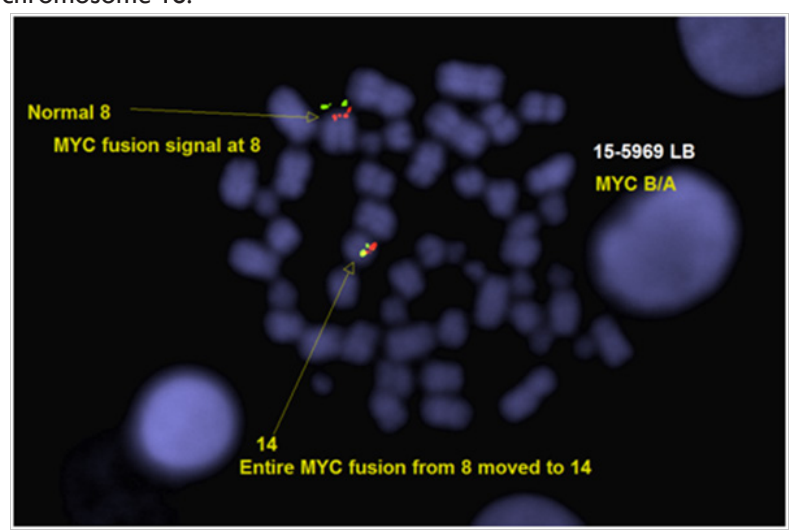

Figure 2B MYC fusion probes show that one copy of the MYC locus has translocated to the derivative chromosome 14 .

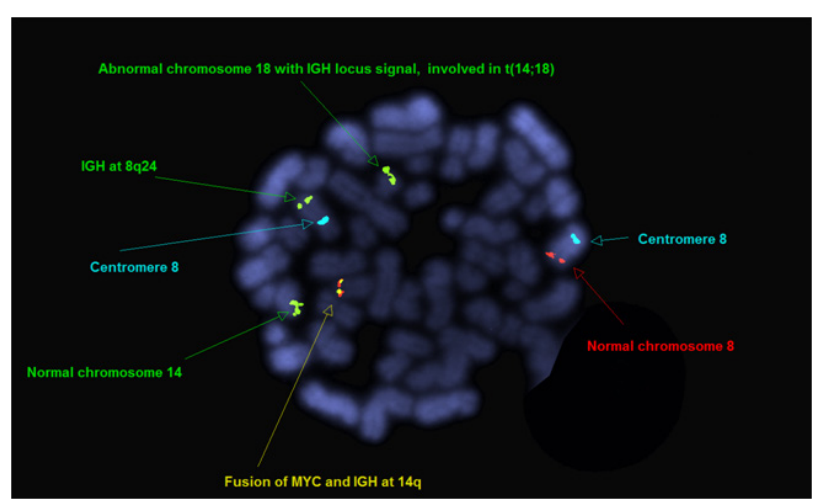

Figure 3 Additional probes reveal a fusion of the entire MYC locus with the residual IGH on the derivative chromosome I4.

\section{Discussion}

The patient's IGH/BCL-2 rearrangement is not unexpected given her long standing history of follicular lymphoma. The complex MYC rearrangement possibly involving the IGH/BCL-2 fusion, however, is unusual, and its precise nature remains unclear. While MYC is commonly involved in double-hit and triple-hit lymphomas, it is unclear whether the current case represents a true break in the MYC locus, or a total insertion of the locus intact. Regardless, the cytogenetic finding of the MYC rearrangement and it's over expression by immunohistochemistry portend a poor prognosis. Other groups have reported complex translocations/rearrangements involving IGH, BCL-2, and or MYC in double-hit B-cell lymphoma arising from follicular lymphoma, ${ }^{4}$ and in large B-cell lymphomas in general, ${ }^{5,6}$ but to our knowledge, the specific insertional translocation of MYC into the der(14) of the $t(14 ; 18)$, and the subsequent movement of the fused IGH/BCL2 to $8 \mathrm{q} 24.1$, has not been previously reported in the literature.

\section{Conclusion}

In summary, we present a case with a novel rearrangement involving MYC in large B-cell lymphoma with an IGH/BCL-2 rearrangement and BCL-2 and MYC over expression by immunohistochemistry.

\section{Acknowledgements}

None.

\section{Conflict of interest}

The author declares no conflict of interest.

\section{References}

1. Ott G, Rosenwald A. Molecular pathogenesis of follicular lymphoma. Haematologica. 2008;93(12):1773-1776.

2. Xu X, Zhang L, Wang Y, et al. Double-hit and triple-hit lymphomas arising from follicular lymphoma following acquisition of MYC: report of two cases and literature review. Int J Clin Exp Pathol. 2013;6(4):788-794.

3. Lindsley R, LaCasce A. Biology of double-hit B cell lymphomas. Curr Opin Hematol. 2012;19(4):299-304.

4. Snuderl M, Kolman OK, Chen YB, et al. B-cell lymphomas with concurrent IGH-BCL2 and MYC rearrangements are aggressive neoplasms with clinical and pathologic features distinct from Burkitt lymphoma and diffuse large B-cell lymphoma. Am J Surg Pathol. 2010;34(3):327-340.

5. Cigudosa JC, Parsa NZ, Louie DC, et al. Cytogenetic analysis of 363 consecutively ascertained diffuse large B-cell lymphomas. Genes Chromosomes Cancer. 1999;25(2):123-133.

6. Zhao X, Fan R, Lin G, et al. Chromosome abnormalities in diffuse large B-cell lymphomas: analysis of 231 Chinese patients. Hematol Oncol. 2013;31(3):127-135. 